\title{
Relevancia de los factores de riesgo laborales en personal de recolección de residuos: una revisión
}

\author{
Sindy Johana Forero Gauna1 (D), Laura Viviana Parra Hurtado² (D, Ángela Liliana Monroy Díaz ${ }^{3}$
}

\section{RESUMEN}

Introducción: En la prestación del servicio público de aseo, los trabajadores se encuentran expuestos a múltiples factores de riesgo que pueden afectar su integridad personal, debido a la ocurrencia de accidentes o enfermedades laborales. Dentro de las actividades que agrupa este servicio se encuentran la disposición, recolección, almacenamiento, transporte y disposición final de residuos; así como el barrido, limpieza de vías, corte de césped o poda de árboles.

Objetivo: Describir los factores de riesgo laborales asociados con la recolección de residuos sólidos urbanos dentro de la prestación del servicio público de aseo tanto en el ámbito internacional como en el nacional.

Metodología: Se llevó a cabo una revisión bibliográfica descriptiva con un enfoque narrativo, usando palabras clave validadas en tesauros, en idioma inglés y español (entre ellas factores de riesgo, ergonomía, residuos sólidos, segregadores de residuos sólidos), y se seleccionaron artículos en un intervalo de tiempo de quince años, para un total de 50 referencias seleccionadas.

Resultados: Los factores de riesgo más estudiados están relacionados con los ambientales, los psicosociales, los biológicos, los mecánicos y los ergonómicos, y dentro de este último el trastorno musculoesquelético es el factor más detectado en los artículos consultados.

Conclusiones: Dentro de los factores de riesgo más prevalentes están los riesgos ergonómicos y el riesgo biológico, teniendo en cuenta las posturas que realizan para la ejecución de las actividades y el contacto directo con los desechos.

Palabras clave: factores de riesgo; ergonomía; residuos sólidos; segregadores de residuos sólidos.

${ }^{1}$ Cootranstame, Yopal, Colombia.

${ }^{2}$ Agata Store, Villa de Leyva, Colombia.

3 Universidad de Boyacá, Tunja, Colombia.

Autora de correspondencia: Ángela Liliana Monroy Díaz. Correo electrónico: almonroy@uniboaca.edu.co

Citar este artículo así:

Forero Gauna SJ, Parra Hurtado LV, Monroy Díaz ÁL. Relevancia de los factores de riesgo laborales en personal de recolección de residuos: una revisión. Rev Investig Salud Univ Boyacá. 2021;8(1):136-151. https://doi.org/10.24267/23897325.564 


\title{
Relevance of Labor Risk Factors in Waste Collection Personnel. A Review
}

\begin{abstract}
Introduction: In the provision of the public cleaning service worldwide, workers are continuously exposed to multiple risk factors that can affect their personal integrity, through the appearance of accidents or occupational diseases, within the activities that the provision groups together. This service includes the disposal, collection, storage, transportation and final disposal of waste, as well as sweeping, cleaning roads, cutting grass, pruning trees.
\end{abstract}

Objective: To describe the occupational risk factors associated with the collection of urban solid waste within the provision of the public cleaning service both internationally and nationally.

Methodology: A descriptive bibliographic review was carried out with a narrative approach using keywords validated in thesauri, in English and Spanish, including risk factors, ergonomics, solid waste, solid waste segregators, articles were selected in a time interval of 15 years, extracting the information from 50 selected references.

Results: The most studied risk factors are related to environmental, psychosocial, biological, mechanical and ergonomic risks, where in the latter the musculoskeletal disorder is the most predominant factor detected in the articles consulted.

Conclusions: Among the most prevalent risk factors are ergonomic risks and biological risk, taking into account the postures they perform for the execution of activities and direct contact with waste

Keywords: risk factors; ergonomics; solid waste; solid waste segregators. 


\section{Relevância dos fatores de risco ocupacional nos trabalhadores de coleta de resíduos: uma revisão}

\section{RESUMO}

Introdução: Na prestação de serviços de saneamento público, os trabalhadores são expostos a múltiplos fatores de risco que podem afetar sua integridade pessoal, devido á ocorrência de acidentes ou doenças profissionais. Entre as atividades incluídas neste serviço estão à eliminação, recolha, armazenamento, transporte e eliminação final de resíduos, bem como varrer, limpeza de estradas, corte de grama ou poda de árvores.

Objetivo: Descrever os fatores de rico ocupacional associados a recolha de resíduos sólidos urbanos na prestação de serviços de saneamento público, tanto a nível internacional como nacional.

Metodologia: Foi realizada uma revisão bibliográfica descritiva com uma abordagem narrativa, utilizando palavras-chave validadas em thesaurus, em inglês e espanhol (entre elas fatores de risco, ergonomia, resíduos sólidos, segregadores de resíduos sólidos), e foram selecionados artigos ao longo de um período de quinze anos, para um total de 50 referências selecionadas.

Resultados: Os fatores de risco mais estudados estão relacionados com fatores de risco ambientais, psicossociais, biológicos, mecânicos e ergonômicos, e dentre estes últimos às perturbações músculo-esqueléticas são o fator mais detectado nos artigos consultados.

Conclusões: Entre os fatores de risco mais prevalecentes estão os ricos ergonômicos e o risco biológico, tendo em conta as posturas utilizadas na execução das atividades e o contato direto com os resíduos.

Palavras-chave: fatores de risco; ergonomia; resíduos sólidos; segregadores de resíduos sólidos. 


\section{INTRODUCCIÓN}

Frecuentemente, los recolectores de residuos sólidos están expuestos a factores laborales que ponen en riesgo su salud, sumado a la alta informalidad de sus trabajos, que incrementan estos riesgos. Debido a ello, es difícil estimar una cifra de trabajadores dedicados a esta ocupación; pero se conoce que millones de familias en el mundo dependen de esta actividad económica.

La Organización Mundial de la Salud menciona que el $50 \%$ de los residuos sólidos generados en Latinoamérica aún recibe una recolección y disposición final inadecuada. Por ello, tales vacíos en la organización de los gobiernos y la poca educación en las comunidades, en relación con la disposición de estos residuos, afectan a los trabajadores encargados de su recolección (1).

En este sentido, la Organización Internacional del Trabajo y Organización Mundial de la Salud mencionan que la salud ocupacional tiene la finalidad de incentivar la prevención y el cuidado de la salud en los trabajadores a través de condiciones, normas y acciones que protejan la integridad de quien labora (1). Así, existen normas como la ISO 45001:2018, cuyo objetivo es especificar los requisitos para el desarrollo de un sistema de gestión de seguridad y salud en el trabajo que orienten a las empresas a brindar un lugar de trabajo seguro y saludable (2). No obstante, tal reglamentación es poco aplicada por el personal encargado la disposición de residuos sólidos y existe un desafío en su implementación y la garantía de cuidado de la salud en estos trabajadores.

En América Latina se estima que se producen hasta 541.000 toneladas al día de basura, pues solo en Bogotá se generan cerca de 6000 toneladas de residuos diarios, que en su mayoría no se gestionan ni se disponen de manera adecuada. Incluso, se espera un incremento de estos residuos hasta en un $25 \%$ para el año $2050(3,4)$. De acuerdo con ello, es posible que aumente la contaminación ambiental y que derivado de ello se afecte la salud de la población en general y de las personas que se dedican a la disposición final de dichos residuos (5), dado que este trabajo se considera una de las diez actividades más peligrosas del mundo (6).

El presente artículo de revisión se justifica, puesto que, en general, los trabajadores están expuestos a diversos factores de riesgo en el ambiente laboral, que varían dependiendo de la actividad desarrollada. Estos riesgos pueden derivar en enfermedades profesionales o accidentes de trabajo, cuando se logra identificar la relación causa-efecto entre el ambiente laboral y la alteración de salud evidenciada (7). Dentro de la gestión de desechos sólidos, en la cual se incluyen actividades que demandan la recolección, el transporte, la disposición, el almacenamiento de 
residuos, así como el barrido y limpieza de vías (8), los trabajadores se encuentran expuestos a 1) riesgos físicos, biológicos, psicosociales y ambientales (por ejemplo: caídas, golpes, cargas pesadas, cortes, atrapamientos, accidentes de tráfico, lesiones cortantes o sobreesfuerzo); 2) a riesgos ergonómicos y psicosociales (por ejemplo: daños posturales, trabajo nocturno, largas jornadas de trabajo), y 3) a riesgos de higiene y medio ambiente (por ejemplo: vibraciones, flujo de aerosoles, químicos, ruido, contacto potencial con agentes biológicos, como la ocasionada por la bacteria Leptospira) $(9,10)$. Los datos que se establecen de estos factores de riesgo son diversos en relación con el país de origen, pues están involucrados con la suficiencia económica de los gobiernos en cuanto a las normas de gestión de residuos, la maquinaria disponible, las capacitaciones orientadas, los salarios, las jornadas de trabajo y la formalidad en su contratación, además de la educación de sus comunidades en la disposición de los residuos.

El objetivo de esta revisión está centrado en identificar los factores de riesgo más prevalentes en personal que se encarga de la recolección de residuos sólidos en el ámbito mundial, con el fin de exponer las problemáticas reportadas en la literatura y que serán la base para investigaciones futuras que busquen minimizar los riesgos identificados, que pueden abordarse desde las diferentes disciplinas en salud.

\section{METODOLOGÍA}

Se llevó a cabo una revisión bibliográfica descriptiva cuantitativa con un enfoque narrativo, mediante la consulta en bases de datos como ProQuest, Psicodoc, Ovid, Legis, Google Scholar y Pubmed, usando palabras clave validadas en los Descriptores de Ciencias de la Salud (DeCS) en idioma inglés y español: factores de riesgo, ergonomía, residuos sólidos, segregadores de residuos sólidos en combinaciones con conectores como AND y OR. La búsqueda se realizó entre agosto y octubre de 2020, con una ventana de quince años, debido al limitado número de investigaciones sobre el tema.

Los criterios de inclusión fueron: fuentes de información nacional e internacional, en idioma inglés y español, que abordaran factores de riesgo en trabajadores encargados de la recolección de residuos sólidos, trabajos en repositorios y normas que orientaran la teoría de los factores de riesgo laborales. Se excluyeron aquellos artículos que no delimitaban las afectaciones de salud en trabajadores de recolección de residuos. Se seleccionaron 78 referentes que cumplían con los criterios de selección. La información se separó en tablas en una base de datos en el programa Excel, donde se identificaron los factores de riesgo más prevalentes descritos en cada manuscrito; posteriormente, se integraron a la presente revisión 50 
referentes, de los cuales 40 fueron evaluados en la sección de resultados.

En cuanto al componente ético, esta revisión se considera una investigación sin riesgo, puesto que se usaron fuentes secundarias de datos para obtener los resultados.

\section{RESULTADOS}

En general, en África se han evidenciado, en mayor proporción, trastornos musculoesqueléticos (TME), especialmente a causa de actividades de tiempo prolongado como levantar, tirar, empujar/cargar o largas caminatas, donde el área más afectada es la espalda baja (11). En Etiopía se describió que en el año el $34 \%$ de los trabajadores, por lo menos, había presentado una lesión ocupacional relacionada con trastornos del sueño y bajos ingresos (12). En India se determinó que hasta el $79 \%$ de los recicladores durante la ejecución de sus actividades ha presentado al menos un TME, sobre todo en la zona lumbar, espalda baja y hombro (13). Adicionalmente, los trabajadores que se encuentran expuestos directamente con los residuos presentan enfermedades de la piel, infecciones oculares y TME (14). En Sudáfrica se determinó que las posturas que los trabajadores realicen en la ejecución de sus actividades influyen en gran medida en el alto nivel de riesgo al cual se pueden encontrar expuestos (15). También en Etiopía se logró establecer que la magnitud de los síntomas de salud ocupacional está relacionada con el uso adecuado de los elementos de protección personal, así como horas de trabajo y experiencia (16).

En Ghana se identificó que las malas actitudes y comportamientos, como no usar equipo de protección personal, la aplicación deficiente de las normas de seguridad por parte de los supervisores y gerentes, el estrés relacionado con el trabajo causado por entornos de trabajo deficiente, la mala remuneración, las malas relaciones laborales y las percepciones negativas de la comunidad sobre el trabajo de los recolectores de desechos domésticos afectan negativamente la salud y seguridad de los recolectores de desechos domésticos (17).

En Corea del Sur los factores de riesgo más comunes se relacionan con trabajadores electrocutados, lesiones por caídas y enfermedades relacionadas con TME, debido posturas inadecuadas (18).

En Hamburgo (Alemania) se identificaron un $67,2 \%$ de dolor de espalda y un $15,4 \%$ de TME (19). El impacto de la ocupación de carga de residuos municipales sobre el desarrollo de TME es muy común también en trabajadores de la India, en quienes oscila entre el $27 \%$ y el $32 \%(20,21)$. En Ankara (Estambul) se determinó que la tasa de dolor de espalda superior fue del $89,1 \%$ en los trabajadores, donde el $41,9 \%$ presentaba dolor de espalda baja y el $80,9 \%$ experimentó dolor de hombro (22). 
Es evidente que en estos países es constante el riesgo de padecer TME. En Malasia e Irán, dichos trastornos obtienen un porcentaje hasta del $92,5 \%$ en los recolectores de residuos, quienes informaron síntomas de, al menos, en una región del cuerpo durante los últimos 12 meses. Las lesiones de la espalda baja y las rodillas fueron más frecuentes y más graves. Las demandas físicas, como levantar la bolsa/balde, tirar/empujar el contenedor de desechos, caminar junto con el carro de la bolsa/balde y saltar hacia arriba/abajo en el camión de basura, y la organización. Dichas demandas fueron los factores de riesgo más importantes para el desarrollo de TME $(23,24)$.

En relación con los estudios enfocados en la investigación de hepatitis $A, B$ y $C$, se encuentra que en Mansoura (Egipto) se investigó la prevalencia de anticuerpos de hepatitis $C$ en los trabajadores y se demostró una prevalencia del $43,3 \%$ de anticuerpos (25). Por otro lado, en Ilorin (Nigeria) se investigó sobre la prevalencia de la hepatitis $\mathrm{B}, \mathrm{y}$ se obtuvo un $16,7 \%$ de prevalencia de anticuerpos (26). En Ática (Grecia) se enfocó el estudio en la determinación de prevalencia de la hepatitis A y se determinó que los trabajadores expuestos presentan mayor prevalencia de anticuerpos que los trabajadores no expuestos directamente (27).

Dentro de los factores de riesgo y su asociación con infecciones parasitarias, se encontró un estudio realizado en Alejandría (Egipto), donde se detectaron 12 especies de parásitos en los trabajadores estudiados, y se determinó que su principal fuente de riesgo es el contacto directo con residuos fecales (28). Adicionalmente, en Banda Aceh (Indonesia) se estudiaron la infecciones helmínticas transmitidas por el suelo y se determinó que el $21,37 \%$ de los trabajadores estudiados estaba infectado por Trichuris trichiura (29). En Cañas y Tilarán (Costa rica) se analizaron cinco agentes biológicos como hepatitis vírica, VIH, tuberculosis y hepatitis. Allí se logró determinar que los trabajadores se encuentran en un nivel de riesgo muy alto, por estar propensos a adquirir cuatro de los cinco agentes biológicos estudiados (30). En la tabla 1 se presenta un consolidado de los factores de riesgos más prevalentes en el personal encargado de la recolección de residuos sólidos.

En Brasil se ha descrito una preocupación hasta del $86 \%$ en los trabajadores de recolección de residuos, derivada de los tipos de vinculación contractual y la renovación de contrato. Se ha determinado que las condiciones laborales son inadecuadas, al ser en muchos casos un trabajo informal que no determina una jornada laboral, lo que impacta en el estrés emocional por la realización de las tareas del hogar y su trabajo al mismo tiempo (31-33). En Perú se encontró que el riesgo psicosocial es elevado, ya que en la cotidianidad laboral se maneja trabajo activo continuo y un nivel elevado en las exigencias psicológicas del trabajador (34). En Colombia se ha 
Tabla 1. Factores de riesgo prevalentes identificados en personal de recolección de residuos

\begin{tabular}{|c|c|c|c|}
\hline \multicolumn{4}{|c|}{ Europa, Asia, África y Oceanía } \\
\hline Clasificación & Tipo de riesgo & Lesiones desarrolladas & Referencia \\
\hline Riesgo ergonómico & Derivado de carga física & $\begin{array}{l}\text { Desarrollo de lumbalgia, fatiga muscular, } \\
\text { fracturas, lesiones osteoarticulares, TME, } \\
\text { dolor de espalda baja y superior }\end{array}$ & $\begin{array}{l}11,13,14,15,1916,20 \\
21,22,23,24\end{array}$ \\
\hline Riesgo psicosocial & Carga mental & Rechazo social & 17 \\
\hline Riesgo mecánico & Caídas, atropellamientos & Lesiones, cortaduras & 18,31 \\
\hline Riesgos biológicos & $\begin{array}{l}\text { Contacto con residuos conta- } \\
\text { minados }\end{array}$ & Leptospirosis, hepatitis B y C & $27,28,29$ \\
\hline Riesgo Ambiental & $\begin{array}{l}\text { Contacto con ambientes conta- } \\
\text { minados }\end{array}$ & Enfermedades respiratorias & $14,16,32$ \\
\hline \multicolumn{4}{|c|}{ Latinoamérica } \\
\hline Riesgo ergonómico & Derivado de carga física & $\begin{array}{l}\text { Desarrollo de lumbalgia, fatiga muscular, } \\
\text { fracturas, lesiones osteomioarticulares, tras- } \\
\text { tornos musculoesqueléticos }\end{array}$ & $38,39,40,41$ \\
\hline Riesgo psicosocial & Carga mental & Rechazo social & $34,35,37$ \\
\hline Riesgo mecánico & Caídas, atropellamientos & Lesiones, cortaduras & $38,39,40$ \\
\hline Riesgos biológicos & $\begin{array}{l}\text { Contacto con residuos conta- } \\
\text { minados }\end{array}$ & Leptospirosis, hepatitis B y C & $42,43,44,45$ \\
\hline Riesgo Ambiental & $\begin{array}{l}\text { Contacto con ambientes conta- } \\
\text { minados }\end{array}$ & $\begin{array}{l}\text { Enfermedades respiratorias, problemas de piel } \\
\text { y problemas digestivos, efectos mutagénicos y } \\
\text { exposición a químicos }\end{array}$ & $40,43,48$ \\
\hline
\end{tabular}

descrito que el $58 \%$ de este tipo de trabajadores trabaja más de ocho horas diarias con un ingreso inferior a un euro por día (35).

Con frecuencia, en Latinoamérica se encuentran lesiones desarrolladas por los trabajadores recolectores de residuos: en Perú se determinó que el $60 \%$ de los trabajadores presentaba dolores musculoesqueléticos en el cuello, el $10 \%$ en el codo y antebrazo, el $35 \%$ presenta riesgo disergonómico leve en el cuello, hombro y columna dorsal (36). Los TME también se presentan en Ecuador, Chile y Argentina, y los factores que llevan a padecer estos trastornos tienen similitudes muy marcadas, debido a las demandas laborales que promueven diferentes alteraciones, 
como dolencias en la articulación de la rodilla, dolor lumbar, dolor de hombro, tobillo y muñeca (37-39).

Con respecto a las fracturas, también se ha descrito que se presentan hasta en un $48,8 \%$, debido a que la medida preventiva sobre los riesgos ocupacionales es inefectiva en un $82,9 \%$ en los trabajadores. Después de la intervención en gestión de seguridad y salud en el trabajo se ha descrito que el riesgo disminuye en un $63,4 \%$. Además, se evidencia en hasta el $94 \%$ del personal el desconocimiento sobre bioseguridad y los riesgos físicos, químicos y biológicos que deben afrontar en su trabajo diario, y del personal que sí tiene conocimientos sobre bioseguridad, en su mayoría, no aplican las medidas de protección, pues se ha descrito que solo el $60 \%$ de los trabajadores usan guantes $(39,40)$. En Chile, los factores de riesgo mecánico son producto del constante movimiento, al igual que el riesgo ergonómico, en el manejo manual de carga, movimiento repetitivo, vibración cuerpo entero y manejo manual de carga, por su elevada exposición cotidiana (41).

Dentro de los riesgos más prevalentes a los cuales se encuentran expuestos los recolectores de residuos urbanos, están los infecciosos o biológicos, debido al contacto directo con material biológico y objetos cortopunzantes contaminados. Algunos de los factores de riesgo estudiados son la transmisión de leptospirosis, hepatitis (A, B y
C), infecciones parasitarias, hepatitis vírica, VIH, tuberculosis, meningitis y helmintos. En Paraguay se investigó la seroprevalencia y los factores de riesgo asociados a la transmisión de leptospirosis y se determinó que de los 339 trabajadores estudiados, el 8,6\% (29 trabajadores) de estos arrojaron un resultado positivo (42). Por otro lado, en Belo Horizonte (Brasil) se investigaron los factores de riesgo asociados con la prevalencia de la infección por el virus de la hepatitis B y C; se encontró que los trabajadores expuestos a los residuos presentan mayor resistencia a contraer hepatitis B con un valor del 9,8\%; mientras que con la hepatitis C se presenta un valor del 3,3\% (43).

En 2017, en Ciudad de Panamá se determinó que los factores de riesgo biológico a los que se encuentra mayormente expuesto el personal que realiza la manipulación directa de desechos sólidos son las salpicaduras en el rostro y las heridas cortantes o cortopunzantes, con valores del $57,1 \%$ y del $62,8 \%$ (44). En 2019, en Arequipa (Perú) se logró determinar que el área de trabajo que mayor riesgo biológico presenta es la de recolección (compactadoras) (45), esto teniendo en cuenta que dentro de la labor se ven involucradas diferentes actividades. En población de Colombia se ha descrito que solo el $13,6 \%$ de los trabajadores está vacunado contra tétano, en su mayoría, y solo el $12,7 \%$ frente a hepatitis B (4648). Así, es un factor de riesgo para el desarrollo de enfermedades infecciosas que la mayoría de los trabajadores no presenten vacunación. 
De acuerdo con la revisión, se determina que, a pesar de la enorme generación de residuos sólidos que a diario se producen, la seguridad y salud en el trabajo de las personas que desempeñan estas labores se ven limitadas por la informalidad en el desarrollo del trabajo, que implica poca exigencia en los esquemas de vacunas o su aplicación, limitación o inexistencia en los esquemas de prevención de accidentes de riesgo laboral enfocados en la ergonomía y escasa investigación o publicaciones desarrolladas en el ámbito nacional colombiano respecto al tema y en estas poblaciones (49). Además, este trabajo que revierte tanta importancia en la salud del planeta se ha relegado a un segundo plano, pues en los gobiernos locales recae la adecuada gestión de estos residuos y el talento humano encargado de esta función (50).

\section{CONCLUSIÓN}

En la población objeto de estudio se evidencia una alta prevalencia de TME, producto de la carga física ejercida en la naturaleza de su trabajo. La exigencia implica movimientos repetitivos y frecuentes con cargas pesadas; además del riesgo psicosocial atribuido en parte al rechazo social y el alto nivel infeccioso de origen viral, parasitario y bacteriano por el contacto con residuos de diversas fuentes. Es prioritario, entonces, llevar a cabo estudios en esta poblaciones que sitúen un diagnóstico respecto a esos factores identificados y acciones que mejoren su calidad de vida.
El estudio presenta limitaciones, por tratarse de una revisión basada en fuentes secundarias de datos y la poca información de investigaciones colombianas al respecto de este grupo poblacional.

\section{AGRADECIMIENTOS}

A la Universidad de Boyacá, por el apoyo en el desarrollo de la revisión.

\section{FINANCIACIÓN}

Este proyecto contó con la financiación de la Universidad de Boyacá.

\section{CONFLICTO DE INTERESES}

Los autores manifiestan que no hubo conflicto de intereses.

\section{REFERENCIAS}

1. Organización Mundial de la Salud. Residuos sólidos [internet]. 2017. Disponible en: https://www.paho.org/es/temas/etras-equipotecnico-regional-agua-saneamiento/residuossolidos

2. Nueva ISO 45001:2018 [internet]. Disponible en: https://www.nueva-iso-45001.com/

3. Sáez A, Urdaneta JA. Manejo de residuos sólidos 
en América Latina y el Caribe. Omnia [internet]. 2014;20(3):121-35. Disponible en: https:// www.redalyc.org/pdf/737/73737091009.pdf

4. Programa de las Naciones Unidas para el Medio Ambiente. Perspectiva de la gestión de residuos en América Latina y el Caribe [internet]. Ciudad de Panamá; 2018. Disponible en: https:// www.magconsultorias.com/wp-content/ uploads/2018/10/UN_Manejo-de-residuosPREVIEW19-ilovepdf-compressed.pdf

5. Flores Rodríguez LE, Giménez Caballera E, Gerlich J, Carvalho D, Radon K. Prevalencia de accidentes de trabajo en trabajadores recolectores de basura en Asunción, Paraguay. 2013-2014. Mem Inst Investig Cienc Salud. 2016;14(2):40-52. https://doi.org/10.18004/ Mem.iics/1812-9528/2016.014(02)40-052

6. López Valdepeña MY, Valle Barbosa MA, Fausto Guerra J. Condiciones laborales y riesgos para la salud en recolectores de basura. Rev Colomb Salud Ocup. 2021;11(1). https://doi. org/10.18041/2322-634X/rcso.1.2021.5898

7. Abella Cuesta JA, Bernal Luna OL. Análisis de identificación de peligros en el trabajo, en 6 de los 16 conjuntos residenciales, en los cuales 35 Colombia SAS presta sus servicios de aseo y mantenimiento [tesis de especialización en internet]. Bogotá: Universidad Distrital
Francisco José de Caldas; 2016. Disponible en: http://repository.udistrital.edu.co/ bitstream/11349/3911/1/Proyecto $\% 20$ de $\% 20$ grado\%20especializaci\%c3\%b3n.pdf

8. Decreto $2981 / 2013$ del 20 de diciembre, por el cual se reglamenta la prestación del servicio público de aseo [Internet]. Departamento Administrativo de la Función Pública [citado 2020]. Disponible en: https://www. funcionpublica.gov.co/eva/gestornormativo/ norma.php?i $=56035$

9. Athanasiou M, Makrynos G, Dounias G. Respiratory health of municipal solid waste workers. Occup Med. 2010;60(8):618-23. https://doi.org/10.1093/occmed/kqq127

10. Lopéz F, Samudio M, Dalva MdA, Águeda C. Seroprevalencia de leptospirosis y factores asociados en trabajadores del servicio de aseo urbano de la Municipalidad de Asunción, Paraguay. Rev Chil Infectol. 2015;32(6):62833. https://doi.org/10.4067/S071610182015000700003

11. Abou-EIWafa, El-Bestar SF, El-Gilany A, et al. Musculoskeletal disorders among municipal solid waste collectors in Mansoura, Egypt: a cross-sectional study. BMJ Open. 2012;2(5):e001338. https://doi.org/10.1136/ bmjopen-2012-001338 
12. Eskezia D, Aderaw Z, Ahmed KY, Tadese F. Prevalence and associated factors of occupational injuries among municipal solid waste collectors in four zones of Amhara region, Northwest Ethiopia. BMC Public Health. 2016;16(1):1-7. https://doi.org/10.1186/ s12889-016-3483-1

13. Singh S, Chokhandre P. Assessing the impact of waste picking on musculoskeletal disorders among waste pickers in Mumbai, India: a cross-sectional study. BMJ Open. 2015;5(9):e008474. https://doi.org/10.1136/ bmjopen-2015-008474

14. Salve PS. A comparative study of prevalence of morbidities among municipal solid waste workers in Mumbai. SN Compr Clin Med. 2020;2(9):1534-42. https://doi.org/10.1007/ s42399-020-00441-7

15. Ncube F, Ncube EJ, Voyi K. Postural analysis of a developing country's municipal solid waste handlers and a reference group of hospital general hands using the RULA method. Global J Health Sci [internet]. 2017;9(10). Disponible en: http://hdl.handle.net/2263/65971

16. Melaku HA, Tiruneh MA. Occupational health conditions and associated factors among municipal solid waste collectors in Addis Ababa, Ethiopia. Risk Manag Healthc Policy.
2020;13:2415-23. https://doi.org/10.2147/ RMHP.S276790

17. Lissah SY, Ayanore MA, Krugu J, Ruiter RAC. Psychosocial risk, work-related stress, and job satisfaction among domestic waste collectors in the Ho Municipality of Ghana: a phenomenological study. Int J Environ Res Public Health. 2020;17(8):2903. https://doi. org/10.3390/ijerph17082903

18. Jeong BY, Lee S, Lee JD. Workplace accidents and work-related illnesses of household waste collectors. Safety Health Work. 2016;7(2):138-42. https://doi.org/10.1016/j. shaw.2015.11.008

19. Velasco Garrido M, Bittner C, Harth V, Preisser AM. Health status and health-related quality of life of municipal waste collection workers-a cross-sectional survey. J Occup Med Toxicol. 2015;10(1):1-7. https://doi.org/10.1186/ s12995-015-0065-6

20. Reddy E, Yasobant S. Musculoskeletal disorders among municipal solid waste workers in India: a cross-sectional risk assessment. J Family Med Prim Care. 2015;4(4). https://doi. org/10.4103/2249-4863.174270

21. Salve $P$, Chokhandre $P$, Bansod D. Assessing musculoskeletal disorders among municipal 
waste loaders of Mumbai, India. Int J Occup Med Environ Health. 2017;30:875-86. https:// doi.org/10.13075/ijomeh.1896.01049

22. Bulduk EO. Work-related stress levels and musculoskeletal disorders among municipal solid waste collectors in Ankara. Work. 2019;63(3):427-33. https://doi.org/10.3233/ WOR-192949

23. Zakaria J, Suadarin EH, Omar FAC, Salleh NFM. Musculoskeletal disorders among municipal solid waste workers. Asia Pac Environ Occup Health J [internet]. 2017;3(1):28-32. Disponible en: http://apeohjournal.org/index. php/v/article/view/41

24. Ziaei $M$, Choobineth $A$, Abdoli-Eramaki M, Ghaem H. Individual, physical, and organizational risk factors for musculoskeletal disorders among municipality solid waste collectors in Shiraz, Iran. Industrial Health. 2018;56(4):308-31. https://doi.org/10.2486/ indhealth.2018-0011

25. El-Gilany A-H, Abou-ElWafa HS, El-Bestar SF, El-Sayer Zaki M. Prevalence of hepatitis $C$ virus antibodies among municipal solid waste collectors in Mansoura, Egypt. Occup Med Health Aff. 2013;1(133):2. https://doi. org/10.4172/2329-6879.1000133

26. Sawyerr HO, Yusuf RO, Adeolu AT. Risk factors and rates of hepatitis $B$ virus infection among municipal waste management workers and scavengers in Ilorin, Kwara State, Nigeria. J Health Pollution. 2016;6(12):1-6. https://doi. org/10.5696/2156-9614-6.12.1

27. Rachiotis G, Tsovili E, Papagiannis D, Markaki A, Hadjichristodoulou C. Are municipal solid waste collectors at increased risk of Hepatitis A Virus infection? A Greek cross-sectional study. Infez Med. 2016;24(4):299-303.

28. Eassa SM, El-Wahab EW, Lotfi SE, El Masry SA, Shatat HZ, Kotkat AM. Risk factors associated with parasitic infection among municipality solid-waste workers in an Egyptian community. J Parasitol. 2016;102(2):214-21. https://doi. org/10.1645/15-782

29. Imansyah Putra $T$, Loesnihari $R$, Panggabean $M$. Soil-transmitted helminth infection and eosinophil levels among waste collectors in Banda Aceh. Indonesian J Tropical Infect Dis. 2018;7(2):27-34. https://doi.org/10.20473/ ijtid.v7i2.7259

30. Centeno Araya MG, Rodríguez Rojas IM, Álvarez Fallas JP. Evaluación de riesgos biológicos en los trabajadores de la recolección de residuos sólidos urbanos ordinarios de las municipalidades de Cañas y Tilarán [tesis de grado en internet]. Universidad Técnica 
Nacional; 2018. Disponible en: http:// repositorio.utn.ac.cr/handle/123456789/158

31. Kulkarni MS, Pingale DP. Effects of occupational exposures on the lung functions and quality of life of garbage collectors in the urban area. Indian J Occup Environ Med. 2019;23(3):102. https://doi.org/10.4103/ijoem.IJOEM_128_19

32. Lee KH, Jung HJ, ParkDU, Ryu SH, Kim B, Ha KC, et al. Occupational exposure to diesel particulate matter in municipal household waste workers. PloS One. 2015;10(8):e0135229. https://doi. org/10.1371/journal.pone.0135229

33. Carvalho $V$, da-Silva $M$, Silva $L$, Borges $C$, da-Silva L, Robazzi M. Riscos ocupacionais e acidentes detrabalho: percepções dos coletores de lixo. Rev Enferm UFPE. 2016;10(4):118593. https://doi.org/10.5205/1981-8963v10i4a11102p1185-1193-2016

34. Popayán Ramírez KA, Vásquez Muñoz F. Riesgo psicosocial en el personal obrero de limpieza pública. Municipalidad Provincial de Barranca, 2018 [tesis de grado en internet]. Huachu, Perú: Universidad Nacional José Faustino Sánchez Carrión; 2018. Disponible en: http://repositorio.unjfsc.edu.pe/handle/ UNJFSC/3164
35. Gómez-Correa JA, Agudelo-Suárez AA, Samiento-Gutiérrez Jl, Ronda-Pérez E. Condiciones de trabajo y salud de los recicladores urbanos de Medellín (Colombia). Arch Prev Riesgos Labor. 2007;10(4):181-7. Disponible en https://archivosdeprevencion. eu/view_document.php?tpd $=2 \& \mathrm{i}=1788$

36. Riquelme Loyola YJ. Factores de riesgo disergonómicos asociado con el dolor músculo esquelético en los trabajadores del servicio de recolección de residuos sólidos de la Municipalidad Distrital de Pillco Marca, Huánuco, periodo diciembre del 2017 a enero del 2018 [tesis de grado en internet]. Universidad de Huánuco, Perú.; 2018. Disponible en: http://repositorio.udh.edu. pe/123456789/1271

37. Kuijer PP, Sluiter JK, Frings-Dresen MH. Health and safety in waste collection: towards evidence-based worker health surveillance. Am J Ind Med. 2010;53(10):1040-64. https:// doi.org/10.1002/ajim.20870

38. Defelippe LA. Las lesiones osteomioarticulares más frecuentes en recolectores de residuos [tesis de grado en internet]. Universidad Fasta Ciencias Médicas, Argentina; 2014. Disponible en: http:// redi.ufasta.edu.ar:8080/xmlui/bitstream/ handle/123456789/636/2014_K_017. pdf? sequence $=1$ 
39. Condori Ayamamani LY. Intervención de enfermería en la prevención de riesgos laborales en trabajadores municipales de limpieza, Juliaca-2014 [tesis de grado en internet]. Universidad Nacional del Altiplano, Puno, Perú; 2014 [citado 2020 nov 5]. Disponible en: http://repositorio.unap.edu.pe/bitstream/ handle/UNAP/1904/Condori_Ayamamani_ Lucia_Yaneth.pdf? sequence $=1$ \&isAllowed $=y$

40. Cárdenas Padilla M, Valladolid Mendoza LE. Prácticas de medidas de bioseguridad y estado de bienestar en trabajadores segregadores de residuos sólidos de la municipalidad provincial de Huancavelica-2018 [tesis de grado en internet]. Universidad Nacional de Huancavelica, Perú; 2018. Disponible en: http://repositorio.unh.edu.pe/bitstream/ handle/UNH/2149/22-.\%20F051_73938849. pdf? sequence $=1$ \&isAllowed $=y$

41. Ramos Ascue JD, Baldeón Quispe W. Análisis de riesgos de la seguridad e higiene ocupacional durante el manejo de residuos sólidos y reciclaje de plástico polietileno. Producción + Limpia. 2017;12(1):63-71. https://doi.org/10.22507/pml.v12n1a6

42. López F, Samudio M, De Assis DM, Cabello Á. Seroprevalencia de leptospirosis y factores asociados en trabajadores del servicio de aseo urbano de la Municipalidad de Asunción,
Paraguay. Rev Chil Infectol. 2015;32(6):62833. https://doi.org/10.4067/S071610182015000700003

43. Mol MPG, Gonçalves JP, Silva EA, Scaponi C, Greco DB, Cairncross S, Heller L. Seroprevalence of hepatitis $B$ and $C$ among domestic and healthcare waste handlers in Belo Horizonte, Brazil. Waste Manag Res. 2016;34(9):875-83. https://doi.org/10.1177/0734242X16649686

44. Quirós Tejedor EM. Riesgos biológicos en la recolección, manipulación y transporte de desechos sólidos [tesis de maestría en internet]. Universidad Metropolitana de Ciencia y Tecnología, Panamá; 2017. Disponible en: http://repositorio.umecit.edu. pa/handle/001/732

45. Dávila Gordillo ZA, Saire Salcedo FY. Evaluación de riesgos biológicos por exposición a los residuos sólidos mediante el método ERBio en los trabajadores de la municipalidad JLBYR-Arequipa [tesis de grado en internet]. Universidad Tecnológica del Perú; 2019. Disponible en: https://repositorio.utp.edu.pe/bitstream/ handle/20.500.12867/2534/Zacarias $\% 20$ Davila_Fabricio\%20Saire_Trabajo\%20 de\%20Investigacion_Bachiller_2019. pdf? sequence $=3$ \&isAllowed $=y$ 
46. Ballesteros V, Cuadros Urrego Y, Botero Botero $\mathrm{S}$, López Arando Y. Factores de riesgo biológicos en recicladores informales de la ciudad de Medellín. Rev Fac Nac Salud Pública [internet]. 2008;26(2):169-77. Disponible en: http:// www.scielo.org.co/scielo.php?script $=$ sci arttext\&pid $=$ S0120-386X2008000200008\&In $\mathrm{g}=$ en\&nrm $=$ iso\&tlng $=$ es

47. Morales-Quispe J, Suárez Oré CA, Paredes Tafur C, Mendoza Fasabi V, Meza Aguilar L, Colquehuanca Huamani L. Trastornos musculoesqueléticos en recicladores que laboran en Lima Metropolitana. An Fac Med. 2016;77(4):357-63. https://doi.org/10.15381/ anales.v77i4.12655

48. Talavera Rodríguez FS, Castillo Herrera B. Higiene y seguridad laboral de los trabajadores del vertedero municipal de la ciudad de Estelí en el II semestre 2015. Rev Cient FAREM-Estelí. 2016;18:85-93. https://doi.org/10.5377/ farem.v0i18.2770

49. Paiva V. Cartoneros, recolección informal, ambiente y políticas públicas en Buenos Aires 2001-2012. Urbe. Rev Bras Gestão Urbana. 2013;5(1):149-58. https://doi.org/10.7213/ urbe.7791

50. Bernache Pérez G. La gestión de los residuos sólidos: un reto para los gobiernos locales. Soc
Amb. 2015;1(7):72-98. Disponible en: https:// www.redalyc.org/pdf/4557/455744912004. pdf

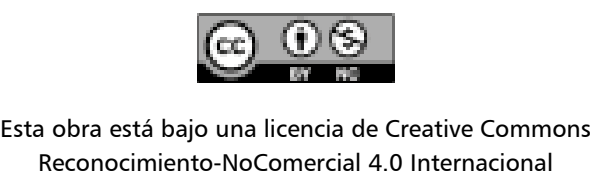

Reconocimiento-NoComercial 4.0 Internacional 